\title{
Septum shadowing by means of a bent crystal to reduce slow extraction beam loss
}

\author{
Francesco Maria Velotti@, Luigi Salvatore Esposito, Matthew Alexander Fraser, \\ Verena Kain, Simone Gilardoni, Brennan Goddard, Michelangelo Pari, \\ Javier Prieto, Roberto Rossi, Walter Scandale, and Linda Susan Stoel \\ CERN, Geneva, Switzerland \\ Francesca Galluccio \\ Università and sezione INFN di Napoli, Naples, Italy \\ Marco Garattini \\ CERN and Imperial College of Science and Technology, London, UK \\ Yury Gavrikov \\ NRC Kurchatov Institute - Petersburg Nuclear Physics Institute, Leningrad, Russia
}

(Received 21 May 2019; published 27 September 2019)

\begin{abstract}
The flux of high-energy protons slow-extracted from the CERN Super Proton Synchrotron (SPS) is limited by the induced radioactivity caused by the beam loss intrinsic to the extraction process. Methods to substantially increase the efficiency of the extraction process are of great interest to fulfill requests for an increasing flux of $400 \mathrm{GeV}$ protons to the present experiments, located in the North Area of the SPS, and also for potential future experiments with very high demanded protons on target. A crystal shadowing technique to significantly reduce the beam scattered and lost on the electrostatic extraction septum during the third-integer resonant slow extraction process has been developed and a prototype system tested with beam. The technique is based on the use of a thin, bent silicon crystal to coherently channel or volume reflect the portion of beam that would otherwise impinge the wire array of the electrostatic septum and instead eject it into the transfer line toward the production targets of the experiments. In this paper, the concept is described and applied to the SPS machine in order to specify the requirements of the prototype crystal shadowing system. Beam dynamics simulations of the prototype system are compared and benchmarked to the results obtained through beam tests, before being exploited to understand the characteristics of the present system and the potential performance reach of an optimized, future operational configuration. The remaining challenges faced to bring the system into operation, the optimization possibilities and other potential applications are discussed.
\end{abstract}

DOI: 10.1103/PhysRevAccelBeams.22.093502

\section{INTRODUCTION AND MOTIVATION}

The slow extraction of a high intensity and energy proton beam suffers in general from the induced radioactivation of the extraction equipment on which the beam unavoidably impinges. With the present technology and extraction efficiency, the attainable annual extracted flux will soon be limited to well below the total flux that the CERN Super

\footnotetext{
*francesco.maria.velotti@cern.ch

Published by the American Physical Society under the terms of the Creative Commons Attribution 4.0 International license. Further distribution of this work must maintain attribution to the author(s) and the published article's title, journal citation, and DOI.
}

Proton Synchrotron (SPS) could deliver because of the impacts of the increased radiation levels in the slow extraction region. The increased failure rate of equipment from radiation-induced damage, combined with the increased waiting times required to access and carry-out hands-on maintenance to reduce dose to personnel, all limit the availability of the machine. Although several possibilities are being investigated, primarily to reduce dose to personnel, including the use of materials in the extraction straight that activate less and extending the use of remote handling techniques during interventions, the focus of recent $R \& D$ efforts has been on tackling the source of the problem and improving the extraction efficiency $[1,2]$. Presently at the SPS, the measured inefficiency of the slow extraction process is on the order of a few $\%$ and its 
reduction is apparently limited by the tolerances and relative misalignment of the long wire-arrays delimiting the low and high field regions inside the electrostatic septa [3].

One of the most promising technological directions to substantially reduce the beam lost during slow extraction is the use of bent silicon crystals for assisting the beam extraction. In particular, the application of a thin, bent crystal aligned upstream of an extraction septum is a novel extraction technique investigated in recent years to significantly reduce the beam intensity impinging the septum $[4,5]$. In literature, one finds examples and discussions of such concepts where a crystal is aligned to a nonresonantly extracted beam upstream of a magnetic extraction septum, but typically in volume reflection. At CERN we took on the challenge of aligning the crystal to both a thin electrostatic septum and the separatrix arm of a resonantly slow extracted beam on the third-integer resonance, testing both the channeling and volume reflection schemes. We refer to this scheme as the 'shadowing' of the septum by the crystal. The shadowing of the SPS electrostatic septum with an upstream thin, bent crystal forms one of the cornerstones of the recent $R \& D$ effort [6].

To put the future proton requests for slow extracted protons at the SPS into context, the SHiP experiment [7], which aims to provide experimental proof for the $\nu \mathrm{MSM}$ theory [8], is requesting an unprecedented flux of $400 \mathrm{GeV}$ protons amounting to an integrated value of $2.0 \times 10^{20}$ on a timescale of 5 years. For this purpose, a short machine cycle of $7.2 \mathrm{~s}$ is foreseen with $4.0 \times 10^{13}$ accelerated and extracted at $400 \mathrm{GeV}$ on a flat-top length of $1.2 \mathrm{~s}$.

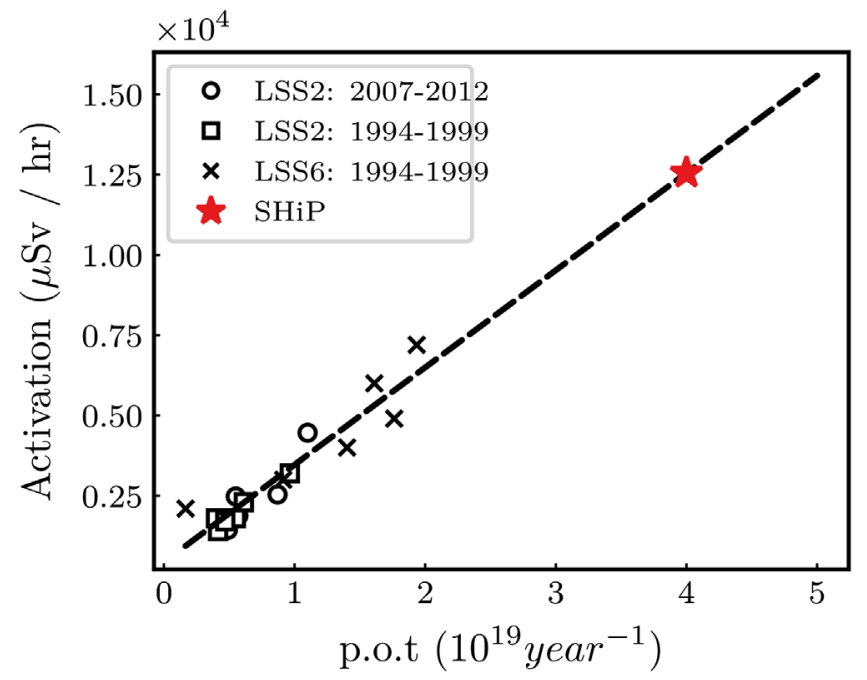

FIG. 1. End-of-run induced radioactivity measured $\sim 30 \mathrm{~h}$ after shutdown at $\sim 1 \mathrm{~m}$ from the beam line over recent years: peak dose rate at electrostatic septum plotted in LSS2 and LSS6. The extrapolation represents the expected activation level for the SHiP scenario should no improvements to the extraction efficiency be implemented.
The accelerated intensity is well within the performance reach of the SPS; however, the annual flux requested of $4.0 \times 10^{19}$ protons is about four times higher than ever achieved for slow extraction through Long Straight Section II (LSS2) to the North Area, as shown in Fig. 1.

\section{SLOW EXTRACTION AT SPS}

Resonant slow extraction is routinely used for delivering long spills over timescales many orders of magnitude longer than the revolution period of the synchrotron. At the SPS a conventional slow extraction technique has been employed for decades where a set of suitably located extraction sextupoles is used to drive resonant amplitude growth in the horizontal plane. In the presence of a large chromaticity, a radio frequency (rf) manipulation is first employed to increase the momentum spread of the beam and further increase the chromatic tune spread, before the extraction is driven and controlled by sweeping the tune of the machine.

The tune is ramped up, from 26.62 to 26.70 , bringing the particles with lowest momentum into resonance before the higher momenta, which then follow later in time, exploiting the beam momentum spread and the machine chromaticity. The tune was conventionally swept by changing the current in the main quadrupole circuits. As a consequence, the optics seen by the on-stopband particles varies as a function of time throughout the spill.

The Steinbach diagram representation of the SPS slow extraction is shown in Fig. 2.

In fact, the separatrix angular spread originates from two different effects. First, particles at the edge of the stopband (largest amplitude and hence maximum momentum deviation from on-resonance particles) will determine the span of the intrinsic separatrix angular spread. Then, as a result of the tune sweep and consequent optics change, the extracted separatrix arm rotates [9]. The variation of the separatrix arm presented to the electrostatic septum during

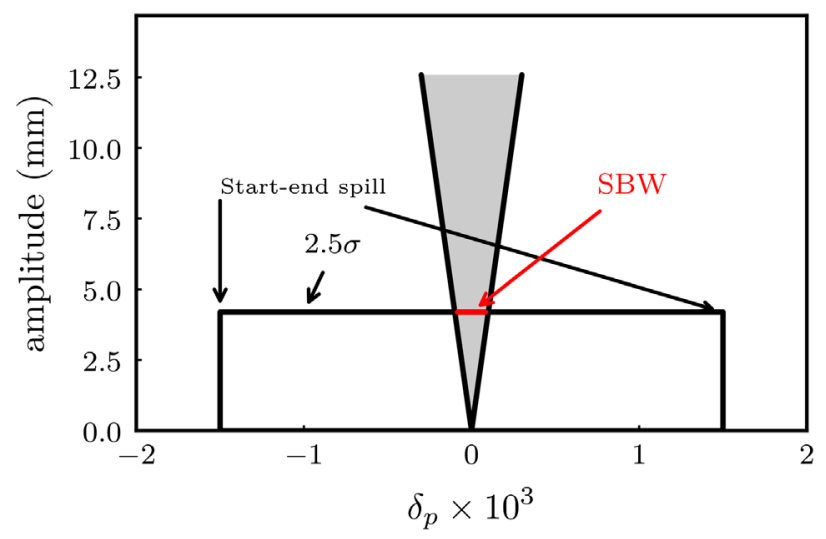

FIG. 2. Steinbach diagram representation of the SPS slow extraction and parameters. The stopband width is approximately $\pm 1 \times 10^{-4}$. 
the extraction process increases the effective angular spread of the beam and the concomitant prompt beam loss. Suppressing this effect was identified at an early stage as a prerequisite improvement needed to successfully channel part of the separatrix arm through the narrow angular acceptance of the crystal [10]. A dynamic bump to control angle and position at the ES, as routinely used in operation at J-PARC [11], was the first option tested in experiment to compensate the separatrix rotation. Although simulations showed promising results and an expected reduction also in the prompt beam loss, the actual implementation turned out to be complex due to feed-down effects as reported in [12]. The problem was finally solved by introducing a new extraction technique where the fields in all SPS magnetic elements are scaled following the momentum distribution of the beam to drive slow extraction and keep the optics of the extracted beam and, hence resonance, fixed. The tune, as calculated in normalized quadrupole strengths, is adjusted to be the resonant one for the on-stopband particle and remains constant. This new extraction technique was named constant optics slow extraction (COSE) and was successfully put into operation during the SPS 2018 run. The details of the technique and implementation can be found in [9]. With COSE the separatrix presentation remains constant throughout the extraction plateau within the precision of the SPS power supplies.

In the case of third-integer resonant slow extraction, particles outside the central triangular stable region grow rapidly in amplitude, returning each three turns to the same separatrix branch and eventually crossing into the highfield region of a well-positioned electrostatic septum, which is typically delimited by an array of wires or a thin foil. The presentation of the beam in phase space at the electrostatic septum in the SPS is shown in Fig. 3 shortly after the spill has started. The electrostatic septum deflects the large amplitude particles out of the circulating beam aperture and into the external transfer line. The beam envelope during slow extraction in LSS2 is shown in Fig. 4, where the largest amplitude particles can be seen leaving the SPS ring. The size of the transverse beam envelope
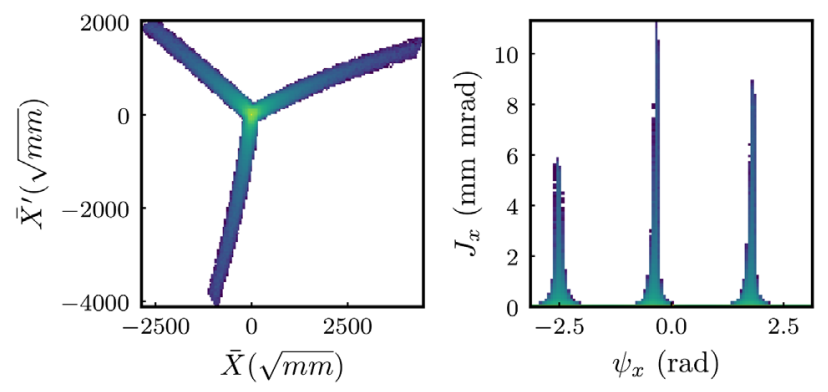

FIG. 3. Simulated normalized horizontal phase space (left) and action-angle variable representation (right) at the electrostatic septum in the SPS about 150 turns after the start of the spill.

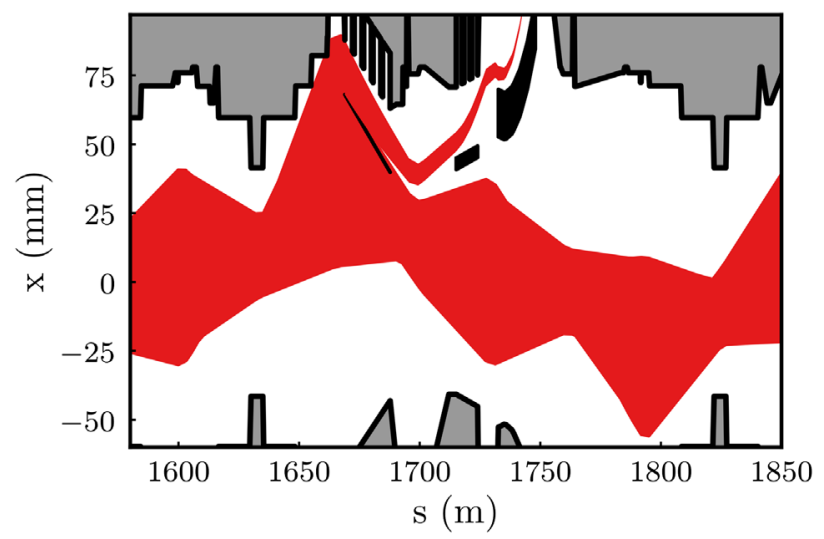

FIG. 4. Simulated beam envelope and machine aperture during slow extraction in the region of the SPS ring around LSS2 for a spiral step of $20 \mathrm{~mm}$.

depends on the maximum three turn amplitude growth, which is represented by the amplitude growth of a particle initially located just inside (circulating beam side of the septum) of the electrostatic septum (ES) before its final three turns in the ring. This amplitude growth at the septum is usually referred to as the spiral step. For the SPS, the operational value is usually in the order of $15 \mathrm{~mm}$, with the design value defining the relevant machine apertures is $20 \mathrm{~mm}$. The upstream transverse position of the ES wires is $68 \mathrm{~mm}$ from the machine magnetic center, as shown in Fig. 4.

The typical spill duration requested by the North Area experiments is $4.8 \mathrm{~s}$, which corresponds to approximately $2 \times 10^{6}$ turns of the synchrotron. For the SHiP experiment's request and for the beam tests of the crystal shadowing concept, a cycle with a $1 \mathrm{~s}$ spill was tested and set-up with the COSE extraction. The chromaticity $\left(\xi_{x}\right)$ is set to -1 and the beam final momentum adjusted by typically $1.5 \times 10^{-3}$ (the rf bucket height) to centre the lowest momentum particles, which are the first to be extracted, on the design closed orbit at the beginning of the flat-top. The extraction sextupoles are set to constant and nominal integrated strength of $k_{2}=-0.089 \mathrm{~m}^{-2}$. Just after reaching extraction energy, a rf manipulation is made to increase and smooth the beam momentum distribution. Such a process lasts around $2.5 \mathrm{~ms}$, as described in [6]. Thanks to this manipulation, the momentum spread distribution is flattened and less sensitivity to mid and high frequency harmonics can be achieved.

The rf is then turned off and beam allowed to debunch. As described above, the magnetic elements are trimmed together to initiate the extraction with a function that uses feed-forward to make the spill rate as uniform as possible [9]. The implementation of COSE is eased at the SPS by the use of LHC Software Architecture (LSA) [13] in its control infrastructure, which allows the trimming of all the machine's power converters with a single high-level momentum parameter. 


\section{BEAM LOSS ON EXTRACTION SEPTUM}

Particles impinge the wire-array composing the extraction septum because of the continuous transverse distribution of the beam and its finite width and length. The LSS2, which hosts the slow extraction system, is the second-worst radioactivated area in the SPS machine with dose rates in excess of several $\mathrm{mSvh}^{-1}$ at a distance of $\sim 1 \mathrm{~m}$ from the beam pipe; only the very localized hot-spot at the beam dump system is higher.

The high rigidity of the SPS proton beam requires around $16 \mathrm{~m}$ of electrostatic septum length, made up of 5 separate septum units which have to be positioned and aligned to the beam independently. The circulating beam passes through the field-free region within the hollow anode support, which is bounded in the direction of the cathode by an array of tungsten-rhenium (W/Re) alloy wires, of $\varnothing 60 \mu \mathrm{m}$ to $100 \mu \mathrm{m}$. The wire-array of 2080 such wires is aligned precisely by the motorization of the anode support to better than $50 \mu \mathrm{m}$.

The extent to which the wire-arrays intercept the beam is determined by the wire material composition and density, wire diameter, straightness, relative alignment, gap field and physical length. The straightness can be warped by thermal effects during extraction dependent on the extraction rate. Particles which impact the wires can be lost via various scattering processes (inelastic and elastic nuclear, energy loss or multiple Coulomb), either locally in the extraction region for large scattering angles, or later in the machine after multiple turns or transfer line for smaller angles. The resulting radioactivation of the equipment occurs through nuclear fission and spallation of the material and the subsequent radioactive decay processes. Therefore, the induced radioactivation for a given machine layout and material composition is directly proportional to the beam loss, which is in turn directly proportional to the number of particles impacting the septum.

The ideal septum has a small thickness, short length, high field and very high straightness and relative alignment precision between the separated wire-arrays. The extraction beam separatrix is also crucial-clearly, the optimum separatrix presentation at the ES for minimum losses is with small phase space angle fully projected in the spatial direction, which does not change from the beginning to the end of the spill and a small angular spread. The extraction separatrix that was indicated in Fig. 3 is simulated with COSE extraction using the SPS beam characteristics for SHiP.

\section{BEAM DEFLECTION WITH BENT CRYSTALS}

The channeling phenomenon of high-energy particles through the lattice of a bent silicon crystal has been experimentally demonstrated both in the SPS and in the LHC [14-16]. The highly ordered atomic structure of crystals gives rise to the channeling effect, where particles that interact with the crystalline structure with a small enough transverse momentum are confined in the potential well between the planes of the crystal lattice. If the crystal lattice is bent, large deflection angles can be achieved in very short distances through the channeling process, allowing for unique accelerator applications, e.g., in collimation [14]. For illustration, to achieve the same $170 \mu \mathrm{rad}$ bend angle in the same length as the $2 \mathrm{~mm}$ crystal used in the SPS tests for the crystal shadowing, a dipole magnet would need a field of $113 \mathrm{~T}$.

The processes of charged particles interacting with the crystal vary significantly. The material is generally amorphous, but in some conditions there are coherent effects, depending on particle charge, momentum and relative orientation between particle direction and crystalline plane direction. The three main regimes in charged particlescrystal interaction discussed for the application of a crystal to shadowing a septum are volume reflection, amorphous scattering and channeling. In a crystal orientation close to the channeling one, one can identify the following regimes as a function of particle deflection: (1) Transition, amorphous, and volume reflection (VR): Particles are scattered along the bent crystal and the output angle is similar to the initial one, except for VR where particles are coherently deflected. The main contributors to such a regime are volume reflection and elastic (or quasielastic) scattering. In case of volume reflection, the mean value of the deflection angle is appropriately equal to the crystal acceptance; (2) Dechanneling: particles trapped in between the crystalline planes can escape them inside the crystal with an intermediate deflection angle between zero and the nominal channeling angle; (3) Channeling: particles are trapped in between crystalline planes until they leave the crystal and thus experience the full deflection angle. The ratio between the total number of particles impacting the crystal and the number of channeled particles is the single-pass crystal channeling efficiency.

As shown in Fig. 5, the probability that different processes occur is a function of the incidence angle of

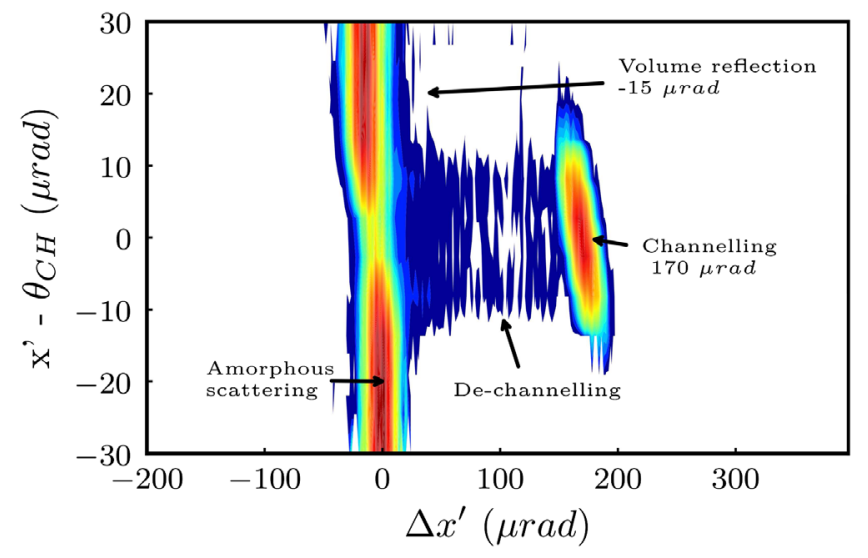

FIG. 5. Intensity crystal response as a function of both deflection angle of the crystal and incoming angle, as measured by the UA9 collaboration [10]. 
the incoming proton with respect to the crystal atomic planes. For the work described in this paper, the angular response of the crystal measured by the UA9 collaboration [10] was implemented in the particle tracking simulations.

\section{CRYSTAL SHADOWING OF EXTRACTION SEPTUM}

A thin bent crystal placed at a suitable location upstream and with a width similar to that of the ES can reduce the number of particles impacting the septum. The crystal can give a large coherent deflection to a substantial fraction of the protons that would otherwise impact the septum, deflecting them past it and into the high-field region to join the extracted particles, as shown schematically in Fig. 6. The loss reduction obtained in this case would be the channeling efficiency balanced by the small increase of losses from nuclear interaction introduced by the crystal.

If the channeling angle and phase advance between crystal and septum is adequate, the coherently deflected protons all miss the septum and can be dumped at a dedicated absorber along the transfer line or directed towards an experimental target. A significant reduction in the particle density at the septum can be obtained with this technique. The larger deflection angle from channeling, compared to volume reflection, means that it can provide a larger separation between the particles impinging the crystal, when in channeling, and those that cross the crystal unperturbed (and hitting the ES wires), increasing the loss reduction at the ES. However, the angular acceptance for channeling is much smaller and the process less efficient. A key requirement is that the extraction separatrix angle of the beam stays within the crystal's channeling acceptance of about $\pm 10 \mu \mathrm{rad}$ (Fig. 5) throughout the spill and from spill-to-spill, which requires a narrow angular spread and excellent control and reproducibility.

Two configurations have been considered for the prototyping of the crystal shadowing concept at the SPS [6]: (i) local —with the crystal located directly upstream of the extraction septum and essentially aligned with it inside the same extraction bump, and (ii) nonlocal-with the crystal located some betatron wavelengths upstream of the

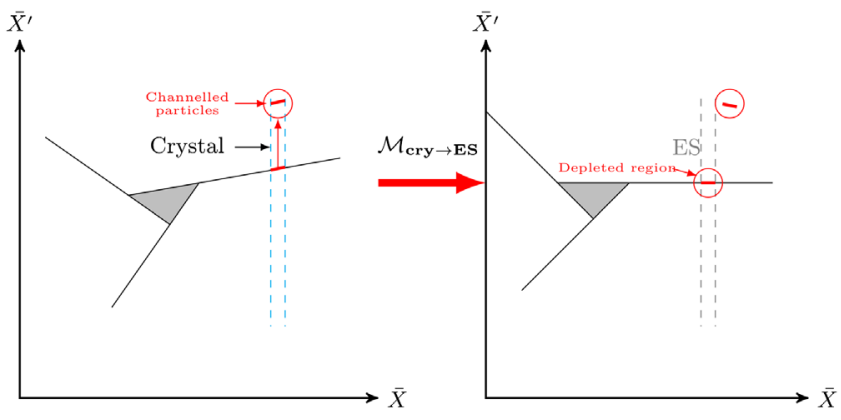

FIG. 6. Schematic representation of third integer resonant slow extraction shadowing of ES with thin crystal. extraction septum and inside a separate closed-orbit bump. The advantage of the nonlocal scheme is that the phase advance from the crystal to ES can be chosen with more freedom. In fact, the detuning with amplitude introduced by the strong nonlinear fields in the last turn(s) in the machine can be exploited to maximize the loss reduction and optimize the emittance of the extracted beam. However, in order to preserve the available acceptance for the injected beam, a second closed-orbit bump would be needed at the crystal, which would complicate operation. In addition, a second region of elevated levels of induced radioactivity would be created next to the crystal located elsewhere in the SPS.

\section{SIMULATION METHODOLOGY}

Comprehensive particle tracking simulations that included the nonlinear beam dynamics of the slow extraction process in the synchrotron, the beam-crystal, and beam-septum interaction were essential to define the required layout, to understand the experimental results and to optimize the performance reach of the concept. The particle tracking code MAD-X [17] was used to define the layout of the SPS with the septa and crystals in the desired locations. The interaction of charged particles with matter, i.e., with the crystal and the septum blade, was included using the scattering routines of pycollimate [6]. The machine parameters were implemented as a function of time to understand the dynamic process of the spill as the tune is swept. The particle tracking was carried out following two approaches: (i) thin lens tracking with MAD-X and (ii) tracking in 4th-order maps obtained with the MAD-X interface to the polymorphic tracking code (PTC) [18]. In this section, the details of the different aspects of the simulation environment are described.

\section{A. Beam-crystal interaction}

The beam-crystal interaction was modeled using an empirical approach and by incorporating the behavior measured by the UA9 collaboration in dedicated beam tests as a 2D probability density function (PDF) in pycollimate. Following this approach, all particles that interact with the crystal are considered escaping it with a change in their transverse momentum according to the thereafter described 2D PDF.

The measured crystal response was transformed into a set of discrete monodimensional histograms as a function of the difference in angle of the incoming beam with respect to the channeling angle of the crystal. The measured data was binned at $5 \mu \mathrm{rad}$ so interpolation was required to produce the PDF reconstructed in Fig. 7. The simulated response using this approach is essentially indistinguishable from the measured data. As the measured data (Fig. 5) were only available in a limited range on incoming angles, the measured 1D PDF at the measured extremes was used 


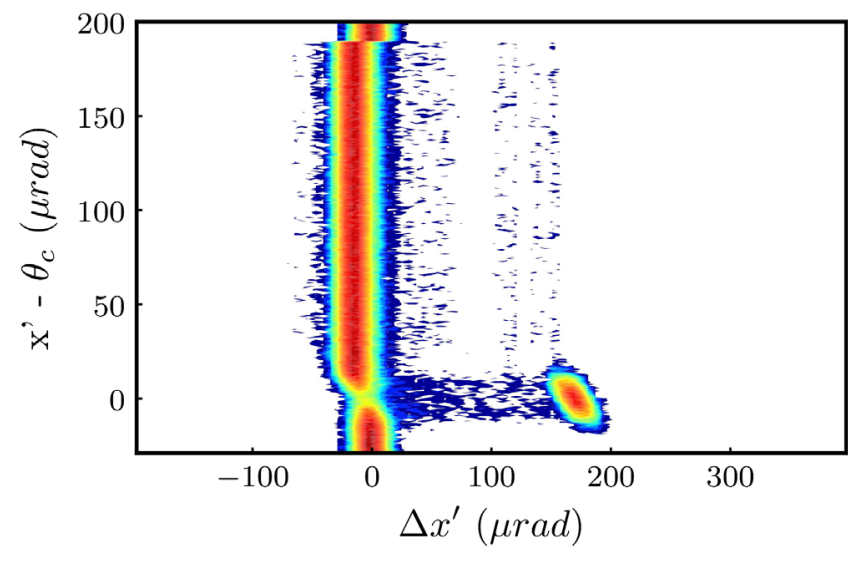

FIG. 7. Rebinned and interpolated crystal scattering response from measurements shown in Fig. 5, as given in input to the tracking simulations.

to extend the angular coverage using theoretical considerations regarding volume reflection, as reported in [19]. It is this PDF, implemented in pycollimate, which is used to calculate the effects of changing the angle between the incoming beam and crystal orientation.

\section{B. Simplified beam dynamics simulations}

A simulation model of the SPS during slow extraction was built with MAD-X as following. The machine tune is set on-resonance for particles on-momentum, i.e., $3 \nu_{x}=80$. The normalized chromaticities are set to their measured values,

$$
\begin{gathered}
\xi_{x}=-1 \\
\xi_{y}=0.58
\end{gathered}
$$

and the extraction bump in LSS2 is turned on. With the implementation of COSE the simulations could be simplified and their speed increased as the optics is theoretically frozen for all extracted particles throughout the spill. It is then only necessary to simulate one instance of the spill, ensuring that the stopband width in relative momentum space $\left(\Delta p / p_{0} \approx \pm 10^{-4}\right)$ either side of the resonant tune is sufficiently covered. The two techniques mentioned above were explored: (i) Slicing the SPS sequence for thin lens tracking with internal MAD-X routines, (ii) Creating the PTC sequence using the MAD-X and extracting maps of the relevant portions of the SPS sequence to a given order.

The first method is pure thin lens tracking through every element of the SPS sequence. When a crystal-type element is encountered the particle distribution is handed over to pycollimate to evaluate the beam-crystal interaction for a given crystal configuration including its position and orientation. This methodology has the advantage of being very accurate, permits aperture restrictions to be rigorously checked and can include time-dependent effects, however it is also timing consuming due to the length of the SPS sequence and the hand-over of the particle distribution between MAD-X and pycollimate at every interaction.

The second method, instead, employs polymorphic maps generated from PTC at a given order to transport the particle distribution from one point to another in the machine. The MAD-X interface allows to chose the integration steps and method used to create the PTC sequence. Four symplectic 4th-order maps were produced, as schematically shown in Fig. 8. In this case, accurate tracking can be performed whilst significantly reducing the computation time, which was essential for the parametric scans needed to assess the performance of crystal shadowing concept presented in this paper.

As the dead time (the time for a circulating particle with a given initial amplitude to jump the ES wires) for a particle with initial amplitude of $1 \sigma_{x}$ is $\approx 100$ turns, the number of turns considered sufficient to simulate the SPS slow extraction dynamics is only 300 . Under this assumption, more than $70 \%$ of the tracked particles are either extracted or lost, with the remaining particles left circulating in the machine. To evaluate the accuracy of the methodology based on tracking in PTC maps extracted from MAD-X, a comparison was performed with PTC tracking (elementby-element) at the 20th order using 150 particles with different initial amplitudes and $\delta_{p}$ ranging $\pm 1 \times 10^{-4}$. The figure of merit chosen to evaluate the accuracy was the normalized variation of the horizontal action $\Delta J_{x} / J_{x}$, where the action is calculated as:

$$
J_{x}=\frac{\bar{X}^{2}+\bar{X}^{\prime 2}}{2}
$$

with $\bar{X}$ and $\bar{X}^{\prime}$ being the horizontal normalized phase space coordinates. The results of the comparison is shown in

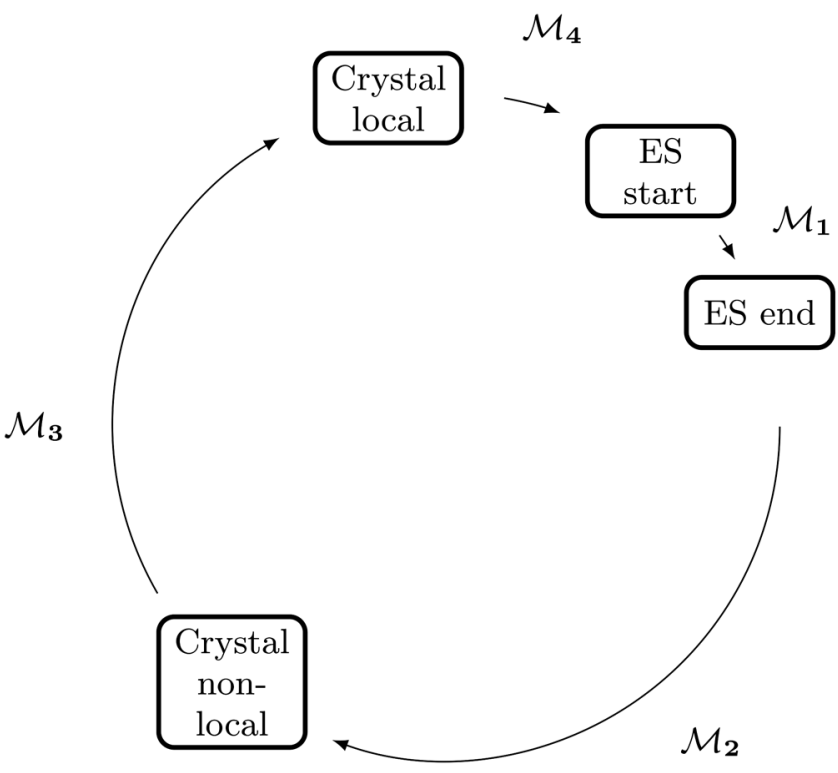

FIG. 8. Schematic view of the SPS maps chosen for tracking. For each sector, a symplectic map at the 4th-order is produced using MAD-X PTC. 


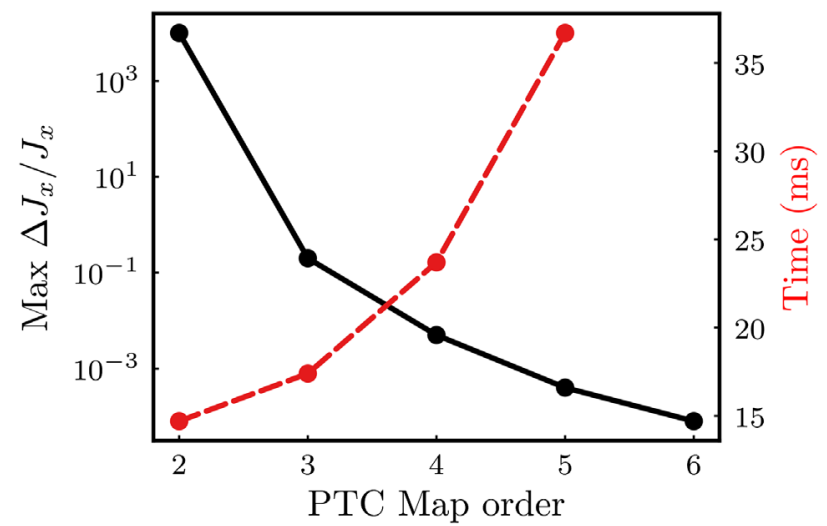

(a)

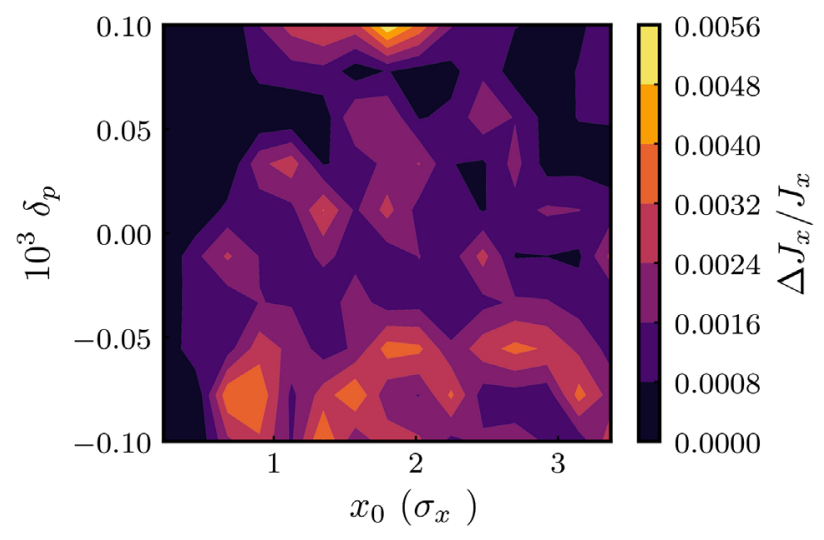

(b)

FIG. 9. Comparison of tracking using sector maps extracted at different order from PTC and element-by-element tracking with MAD-X PTC at 20th-order, using 150 particles tracked for 300 SPS turns. (a) Maximum error on the horizontal action (black) and time needed for tracking (red) as a function of the map order. (b) Maximum error on the action using 4th-order sector maps with different initial conditions.

Fig. 9(a) where a benchmark is presented using the SPS lattice configured for slow extraction. Clear convergence is observed as the map order is increased. Due to the time needed for very high-order maps, 4th-order maps were chosen as good compromise between accuracy and computational time. In fact, the maximum error between pure thick tracking element-by-element using PTC and sector maps at 4th order, for the lattice configuration under analysis, is less than $0.6 \%$ in horizontal action, as shown in Fig. 9(b).

\section{LOCAL SHADOWING}

A suitable location for the crystal in LSS2 was identified 5 $\mathrm{m}$ upstream of the ES separated by a focusing quadrupole (QFA.21610) and $4^{\circ}$ of phase advance. The small phase advance from the crystal to the ES is still adequate to demonstrate the shadowing concept. Using the simulation tools described in the previous section, tracking simulations were performed to specify the crystal characteristics needed to achieve loss reduction at the ES. In the simulation studies presented in this paper an effective ES thickness of $500 \mu \mathrm{m}$ was assumed based on the results of empirical linear scans of the passive (wire-array) diffuser installed and tested with beam earlier in 2018 [20]. The parameters of the crystal used for the SPS tests were specified in [21]. In order to more accurately simulate the beam tests of the shadowing concept in the SPS, the features of the installed crystal are included by modifying the 2D PDF implemented in pycollimate and its thickness. In Fig. 10, the simulated phase space presentation at the crystal [Fig. 10(a)] and ES [Fig. 10(b)] is shown. As expected, when the crystal is aligned and orientated with the separatrix such that it channels particles arriving at a horizontal position shadowing the downstream ES, a local density reduction at the wires of the ES can be observed. When the crystal is aligned in volume reflection a density depleted region is also formed, with particles being kicked back into the machine to circulate another three turns, before being extracted as part of the tail at large amplitude on the extracted beam. The loss reduction factor for the local

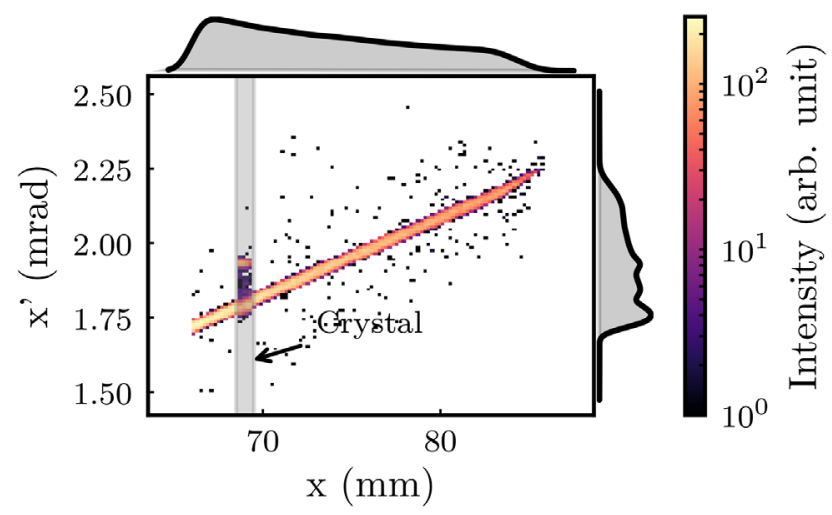

(a)

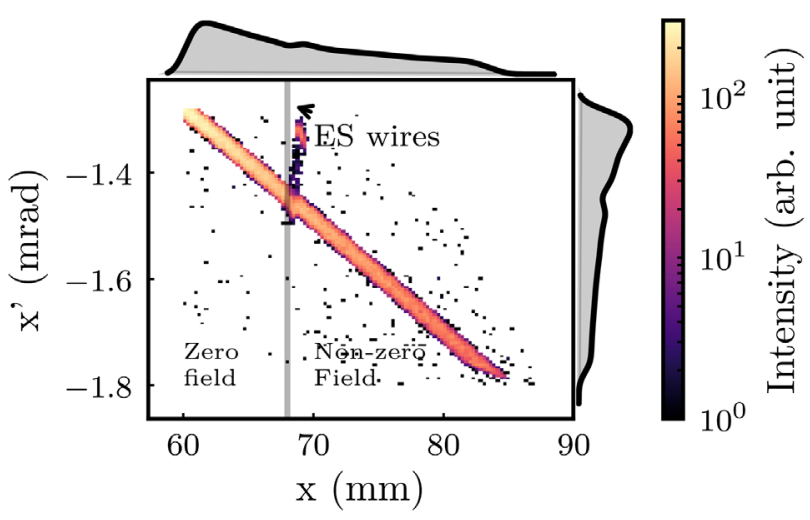

(b)

FIG. 10. Shadowing of ES with thin crystal. The phase space distribution at the crystal of width $0.8 \mathrm{~mm}$ (shaded) with the coherently channeled particles is plotted, together with the phase space distribution at extraction septum with particles missing the thin wire-array (shaded) on the high-field side and transported into the extraction line. (a) Crystal. (b) Electrostatic septum. 


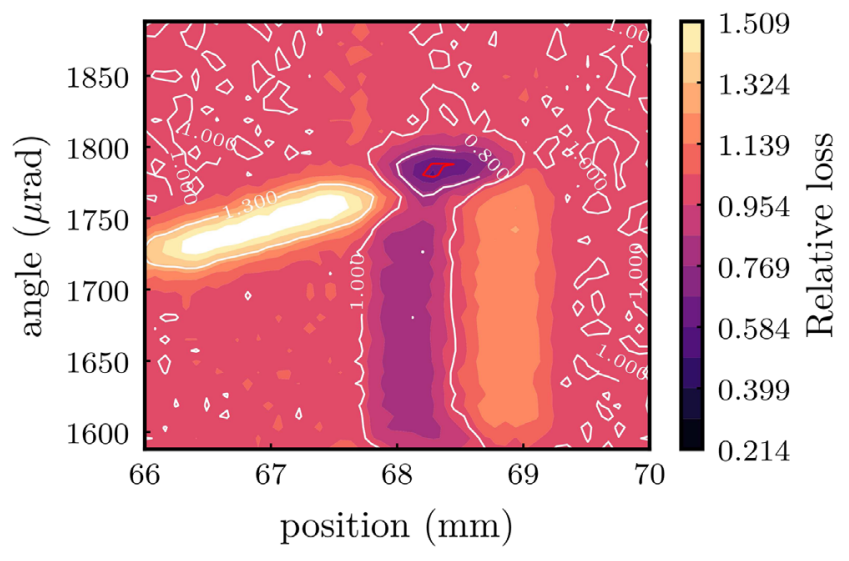

(a)

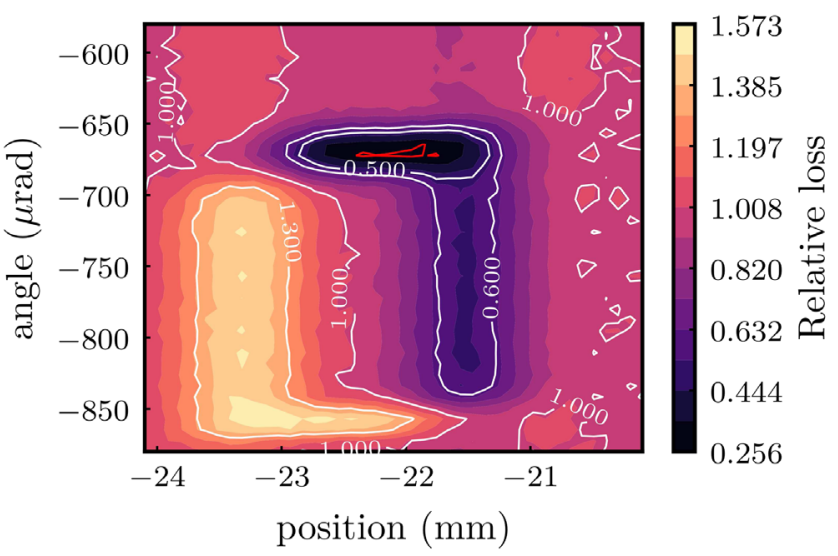

(b)

FIG. 11. Relative beam loss during extraction for the two scenarios studied in simulation: a local crystal in LSS2 and a nonlocal crystal in LSS4. For this scans the ES effective thickness has been assumed of $500 \mu \mathrm{m}$ and being angularly aligned with the separatrix. (a) Local shadowing of the ES with a bent crystal: $0.8 \mathrm{~mm}$ thick and with $174 \mu \mathrm{rad}$ deflection in channelling. The red contour represents loss reduction of $40 \%$ with respect to normal slow extraction losses (no crystal). (b) Non-local shadowing of the ES with a bent crystal: $1.8 \mathrm{~mm}$ thick and with $174 \mu \mathrm{rad}$ deflection in channelling. The red contour represents loss reduction of $75 \%$ with respect to normal slow extraction losses (no crystal).

shadowing case is shown fully parametrized in Fig. 11(a), as a function of both crystal transverse position and angle. The area marked with a red circle represents the global minimum.

The maximum loss reduction when the crystal is shadowing and channeling is close to a factor of 2 , at a reduction of $42 \%$ with respect to the losses recorded with the crystal in a misaligned, amorphous regime. When shadowing and aligned in VR the crystal provides a loss reduction of approximately $20 \%$. It is interesting to note the regions of elevated loss levels at the ES when the crystal is misaligned and a beamlet of particles is either channeled or volume reflected directly onto the wire-array composing the septum blade.

\section{NONLOCAL SHADOWING}

The closed-orbit correctors (COD) in the SPS are installed at every quadrupole (horizontal at QFs and vertical at QDs), however their maximum strength is sufficient to correct the orbit of the circulating beam only up to about $250 \mathrm{GeV} / c$. As the SPS slow extraction is performed at $400 \mathrm{GeV} / c$, local orbit variations using the machine CODs are not possible. At high energy, for both fixed target and LHC beams, strong corrector dipoles, the so-called bumpers, are used to induce local variations in the closed-orbit to reduce the distance between the circulating beam and blade of the extraction septum. Three LSS are equipped with bumpers, LSS2 (as described before), LSS4 and LSS6. To preserve the acceptance for the injected beam and hence allow operation with a stationary crystal in the normal SPS cycling mode, the choice for a nonlocal position for the crystal in the SPS was restricted to one of these LSS regions.

The location with enough space to host a goniometer tank inside the region of a closed-orbit bump and with a phase advance of $\Delta \mu_{\text {cry } \rightarrow \mathrm{ES}} \sim n \times \pi$ was found to be in the AWAKE and LHC extraction region of LSS4, in close proximity to the fast extraction septa. At this location, the phase advance condition has $n$ odd and this represents an additional advantage for the concept, as the crystal would then be actuated from the inside of the machine, which is opposite to where the SPS fast extraction elements are installed, giving more aperture for the fast extracted beam in LSS4.

Using the simulation tools and approach described above, particle tracking simulations were carried out to evaluate the performance reach of the nonlocal concept, as already shown for the local case. The expected phase space at the crystal (Fig. 12(a)) and at the ES (Fig. 12(b)) are shown in Fig. 12. A clear depletion in particle density can be observed at the transverse coordinate of the ES. The first difference with respect to the local case is that the effect of the crystal is magnified when a larger portion of the SPS is put between the crystal and the ES. The difference of linear phase-advance between crystal and ES for both the local and nonlocal case is (i) Local: $\Delta \mu_{\text {cry } \rightarrow \mathrm{ES}}=4^{\circ}$ (ii) Nonlocal: $\Delta \mu_{\text {cry } \rightarrow \mathrm{ES}}=(n \times 180)+12^{\circ}$.

In fact, there is very little difference in the linear phase advance (as both are very close to multiple integer of $180^{\circ}$ ) between the two cases, however a significant difference in the effect on the crystal shadowing concept can be seen. This effect is caused by the nonlinear fields located between the crystal in LSS4 and ES in LSS2, and especially the two extraction sextupoles (one in LSS5 and one in LSS1), which are encountered by the channeled beam in the last turn. Although the thickness of the crystal needed for proper shadowing depends on the effective thickness of the ES, the aforementioned nonlinear effects also play a significant role in the required thickness of the nonlocal crystal. For the nonlocal case a significantly thicker crystal of $1.8 \mathrm{~mm}$ is required in order to obtain the maximum loss reduction. The thickness is determined by the extremities of the separatrix 


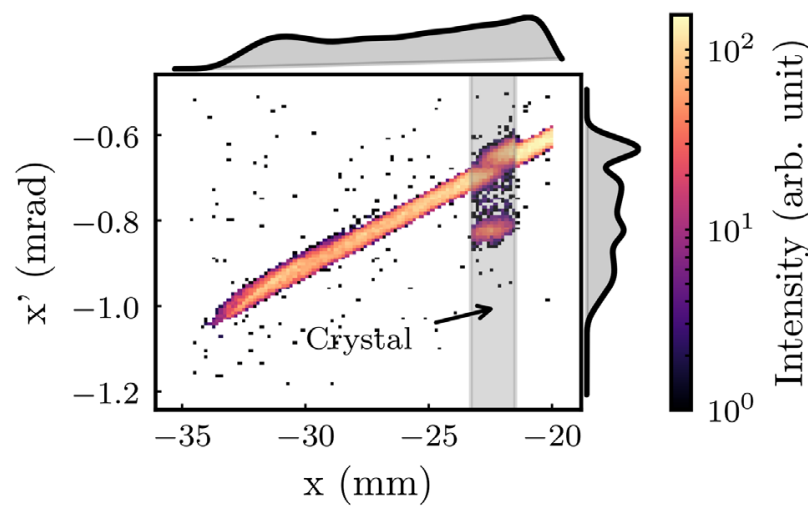

(a)

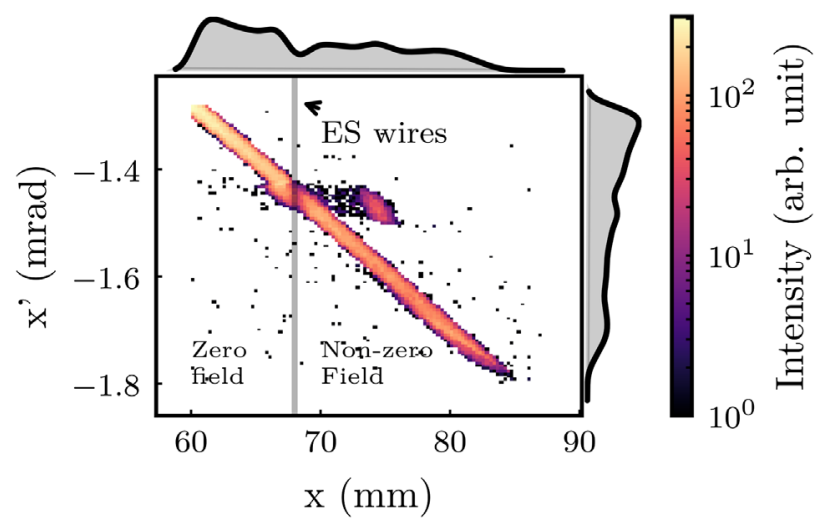

(b)

FIG. 12. Shadowing of ES with thin crystal. The phase space distribution at the crystal of width $1.8 \mathrm{~mm}$ (shaded) with the coherently channeled particles is plotted, together with the phase space distribution at extraction septum with particles missing the thin wire-array (shaded) on the high-field side and transported into the extraction line. (a) Crystal. (b) Electrostatic septum.

formed by the cut made by the crystal location and how they rotate and are presented to the ES. In Fig. 13 the loss reduction achievable with nonlocal shadowing is plotted parametrically as a function of crystal and ES width. For a

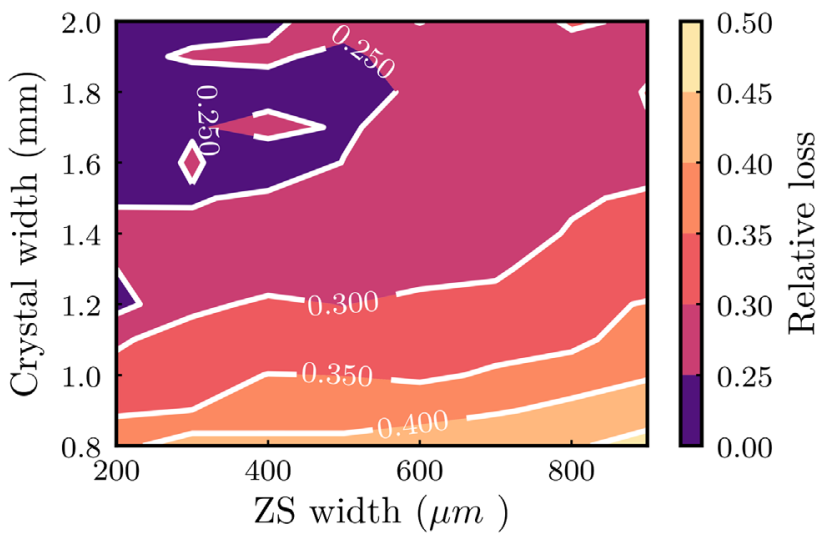

FIG. 13. Relative beam loss for nonlocal scenario parametrized as a function of the crystal and ES thickness. septum with effective thickness up to about $500 \mu \mathrm{m}$ a crystal of $1.8 \mathrm{~mm}$ can produce a loss reduction up to a factor four.

In Fig. 11(b), the simulated loss reduction for the nonlocal shadowing concept is shown parametrized as a function of the crystal position and angle. As expected, when the crystal is positioned correctly to shadow the ES and aligned to channel, a factor of four reduction in loss at the ES is achieved. When the crystal is aligned in VR, almost a factor two reduction can be expected. This is a significant improvement on a crystal located immediately upstream of the ES.

A further possible improvement to the nonlocal concept is to find a solution where the amplitude detuning from the nonlinearities experienced by the beam in the last turns in the SPS can be fully exploited to reduce even more the effective phase space area occupied by the extracted beam, i.e., the extracted beam including the channeled beamlet. One proposal is to place the crystal even further and accommodate another extraction sextupole between the crystal and the ES to amplify this effect. This should significantly simplify the transport of the extracted beam.

\section{SPS BEAM TESTS}

The prototype crystal diffuser tank developed by the UA9 collaboration for this application [19] was installed in the same location as a prototype passive (wire-array) diffuser [20], which was swapped out during the second Injectors Technical Stop in September 2018. The longitudinal space available on the beam line was limited to $180 \mathrm{~mm}$. The crystal is $2.5 \mathrm{~mm}$ long (in the direction of the beam), $0.8 \mathrm{~mm}$ thick (across the beam) with a $174.4 \pm 0.1 \mu \mathrm{rad}$ bending angle and is mounted on a holder with a vertical opening of $35 \mathrm{~mm}$ and a $35.8 \mathrm{~mm}$ clearance from the working crystal surface to the metal support. The crystal was orientated such that the channeled particles are deflected towards the outside of the ring. The installed crystal was chosen conservatively thicker than the effective thickness of the ES measured with the passive diffuser to give some margin in aligning the system for the first time. The holder is mounted inside a tank equipped with a goniometer, which is needed to allow the correct angular alignment of the crystal with respect to the incoming beam. This is important as charged particle deflection with good efficiency is achieved only when the crystal lattice is aligned within $\sim 10 \mu \mathrm{rad}$ of the trajectory of the particles (at $p=400 \mathrm{GeV} / c$ ).

In October 2018, the prototype crystal goniometer was tested with $400 \mathrm{GeV} / c$ protons at an intensity of approximately $5 \times 10^{12}$ protons per cycle, parasitically to the dedicated SHiP prototype target tests which totaled about 20 hours of beamtime and $\sim 6000$ extractions. The shortened cycle with a $1.2 \mathrm{~s}$ flat-top was used for the test with COSE employed. The beam tests were characterized by the following observables using the available instrumentation: (i) Beam loss signal recorded at the LHC-type BLM installed next to the crystal. This BLM could independently identify when the crystal was aligned to the beam and 
channeling. (ii) Sum of the 5 beam loss signals recorded at all SPS-type BLMs installed along the ES and an additional one located immediately downstream. This signal, when normalized to the extracted intensity was used to assess the loss reduction achieved. (iii) Encoder readings of the motors driving the goniometer position and angle. The transverse position of the crystal and its angle could be inferred from readings of the two motors (iv) Time evolution of losses recorded using LHC-type BLM installed at the ES. (v) Beam profile monitors, most notably the wire grid located immediately upstream of the ES.

As the goniometer tank was prealigned with the SPS axis the crystal could be well-positioned in front of the ES. With the crystal inserted into the edge of the extracted separatrix, a first angular scan of the crystal's orientation was performed and the beam loss observed on an LHC-type beam loss monitor (BLM) installed immediately downstream of the goniometer. The large dynamic range, sensitivity and fast-readout of the LHC BLM allowed a clear signal of channeling to be identified with a significant reduction in the beam loss on the crystal. Once the channeling angle was found, the transverse position was varied and successive angular scans performed. The position was changed with the aim of finding the correct transverse location to achieve shadowing of the downstream ES. The range of the angular scans were adapted as the transverse position changed using the theoretical linear projection of the separatrix in phase space.

Shadowing was most clearly identified when the crystal position motor read $70 \mathrm{~mm}$ and its angle was scanned, as shown in Fig. 14. The two regimes that provide loss reduction at the ES via shadowing are visible in the angular scan of Fig. 14: (i) channeling, with a loss reduction recorded of $40 \%$ to $44 \%$ (depending on the angular step) and (ii) volume reflection, with a loss reduction in the order of $20 \%$ across a much wider angular acceptance of $\sim 170 \mu \mathrm{rad}$. The agreement with simulation is within the

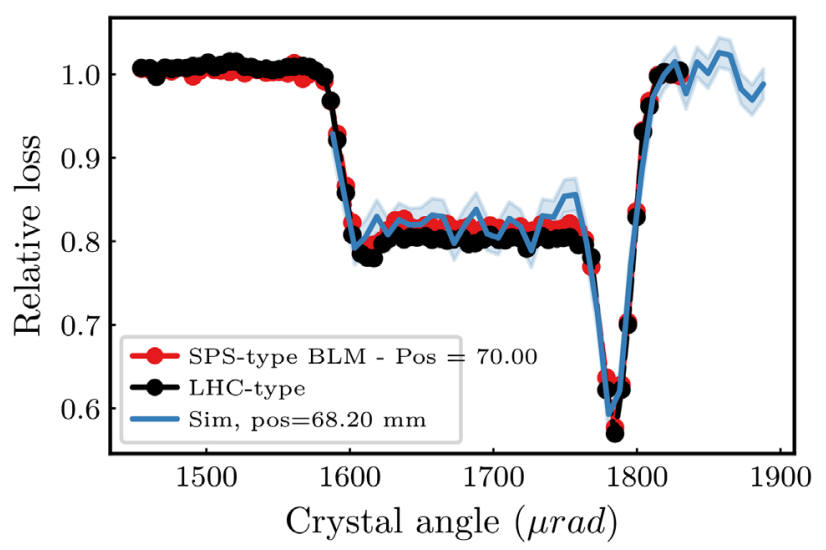

FIG. 14. Relative beam loss measured on the BLMs next to the ES during an angular scan of the crystal with it positioned to shadow. The horizontal axis is expressed in MAD-X reference system. statistical errors of the simulation and the uncertainty of the measurements. The maximum loss reduction recorded when the crystal was channeling stands in good agreement with prediction from tracking simulations that assume an effective ES thickness of $500 \mu \mathrm{m}$.

Repeated angular and linear scans were performed in order to find the global optimum of the loss reduction. These measurements are summarized in Figs. 15 and 16 where the measured loss reduction factor is shown in comparison to simulation with the crystal aligned in channeling and volume reflection, respectively.

When the crystal is positioned to the inside of the ES, towards the circulating beam, the channeled particles are deflected directly into the ES wires, at a longitudinal location that depends on the transverse offset and deflection angle. This increases the beam loss at the ES and is well understood and identified by the high-loss region shown in the parametric simulation studies of Fig. 11(a). When the crystal is to the outside of the optimum position, the channeled particles are extracted but the ES is no longer shadowed and so the overall losses increase slightly due to losses from nuclear interaction at the crystal.

The opposite effect is seen for volume reflection because the angle of deflection has the opposite sign, deflecting protons back into the machine. When the crystal is positioned towards to the outside of the ES, toward the extracted beam, the volume-reflected particles are deflected back toward and directly into the ES wires. This increases the beam loss at the ES. Otherwise, particles are scattered into the machine and circulate another three turns, before being extracted as part of the tail at large amplitude on the extracted beam. In this case the ES is shadowed and the beam loss is reduced.

The most challenging aspect is to simulate the BLM response when the channeled beamlet impacts the ZS

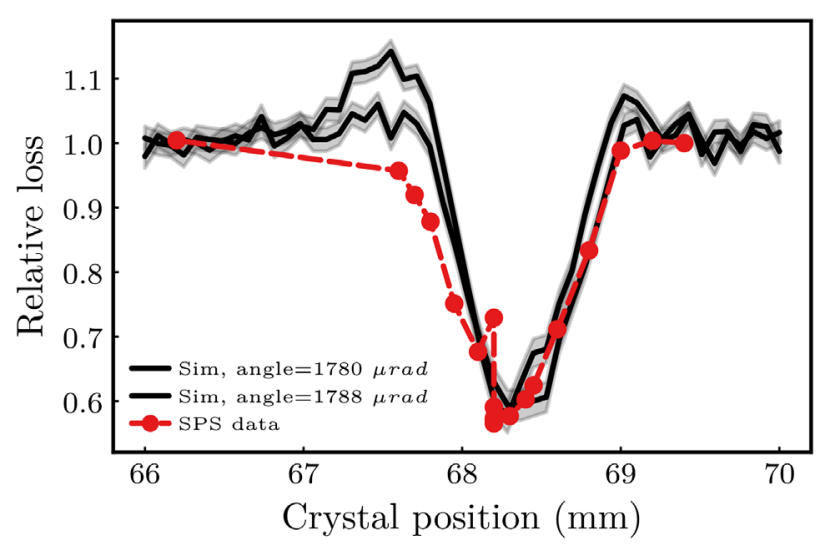

FIG. 15. Relative beam loss measured on the BLMs next to the ES as a function of the crystal position when aligned to the beam in channeling. Simulations for different angles are plotted to show the fluctuations in losses with the chosen angle, as this contributes to the uncertainty in the prediction of the loss reduction. The horizontal axis is expressed in MAD-X reference system. 


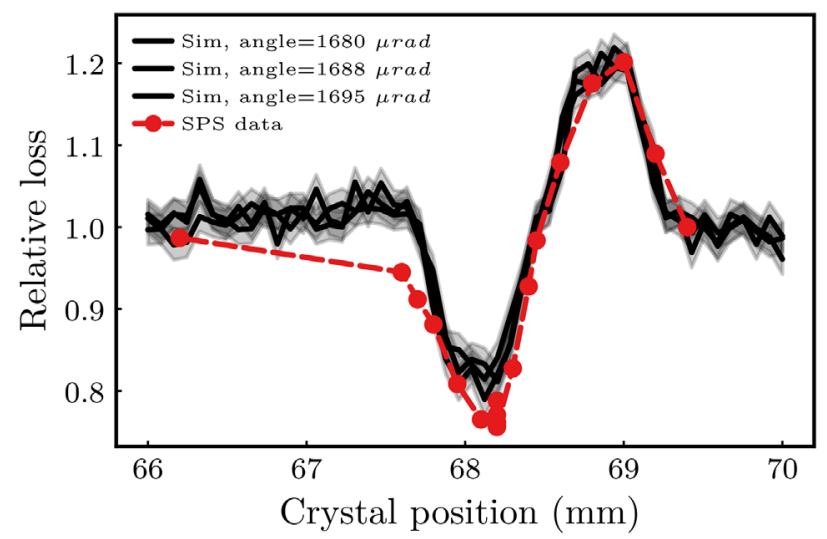

FIG. 16. Relative beam loss measured on the BLMs next to the ES as a function of the crystal position when aligned to the beam in volume reflection. Simulations for different angles are plotted to show the fluctuations in losses with the chosen angle, as this contributes to the uncertainty in the prediction of the loss reduction. The horizontal axis is expressed in MAD-X reference system.

directly along its length. This is challenging because the losses are very localized and hence very difficult to predict exactly the BLMs response without detailed and intensive simulations. In addition, in this case the details of the exact alignment of individual septa tanks becomes important and the simulations consider only the summed number of particles hitting the ES wire-arrays, which are modelled as a single monolithic block. Nevertheless, the general agreement is impressive when considering all the possible sources of uncertainty coming from the measurement systems and from the complex geometry, extraction dynamics, and beam-matter interactions.

It is worthwhile to point out an important observation that the transverse positions corresponding to the minima of the beam loss for shading in both volume reflection and channeling coincide within $0.1 \mathrm{~mm}$. This means that a single linear scan in volume reflection can be used to establish the optimum crystal position, before a single angular scan is needed to establish channeling. This is important for a quick and easy operational setting up of the shadowing system and to avoid the necessity of scanning a relatively large parameter space in both position and angle.

An important information that can be retrieved from the comparison between measurements and simulations is the effective thickness of the ES. The increase in losses shown in the VR position scan (Fig. 16) is essentially a profile of the ES. The thickness estimated in this way is $500 \mu \mathrm{m}$, which is in line with what has been obtained from the analysis, combined with simulations, of the diffuser data [20].

Indeed, the disentanglement between the contribution to the ES effective width from mechanical misalignment and separatrix angular spread shall be assessed. In the previously discussed comparison and width estimation, it was observed that the mechanical misalignment of the ES is the dominant source. The crystal channeling acceptance can be exploited to directly quantify the separatrix angular spread. In fact, when the different parameters were varied to match simulations to measurements, for the situation presented in Fig. 14, the simulated angular spread that better represents the data was found to be $10.5 \mu \mathrm{rad}$ (RMS). This is in very good agreement with theoretical expectations, hence the missing contribution to reproduce the position scan in VR (Fig. 16) was the mechanical ES misalignment.

\section{A. Channeled beamlet trajectory in TT20}

The channeled beamlet could be observed jumping the ES wires, along with a depleted region of density, on a wire-grid profiler located immediately upstream of the ES, as shown in Fig. 17.

Beam loss monitors installed in the extraction transfer line were crucial to help identify where the channeled beamlet grazed the aperture and to help resteer the trajectory of the beam in the transfer line towards to the SHiP prototype target. The trajectory of the channeled beam compared to the nominal beam's envelope is shown in Fig. 18. The location of the observed beam loss matched the locations foreseen from simulation. Resteering the line to optimize the acceptance removed the beam loss and allowed the beam tests to progress without perturbation to the SHiP prototype target test.

For the operational system, the channeled beamlet needs to be properly handled in the transfer line, to avoid local activation or even damage, given the high intensity in the relatively small spot size determined by the crystal width and vertical beam size. Different solutions are being investigated. For example, a collimator in TT20 could be foreseen to absorb the channeled beam and avoid uncontrolled losses. Also, a transport optics which accounts for the channeled beamlet is under deployment and this could represent the fastest and easiest solution to deploy crystal

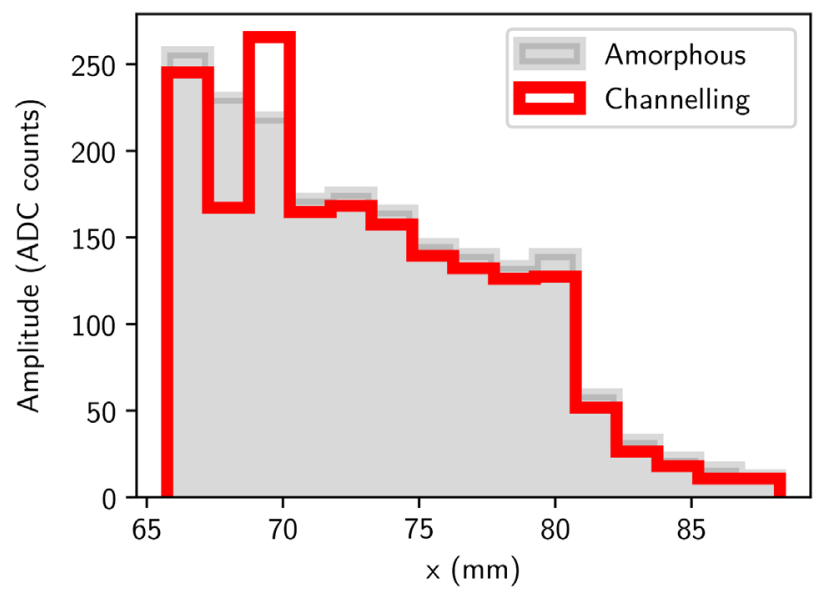

FIG. 17. Horizontal beam intensity distribution measured on a wire-grid beam profiler located immediately in front of the ES. 


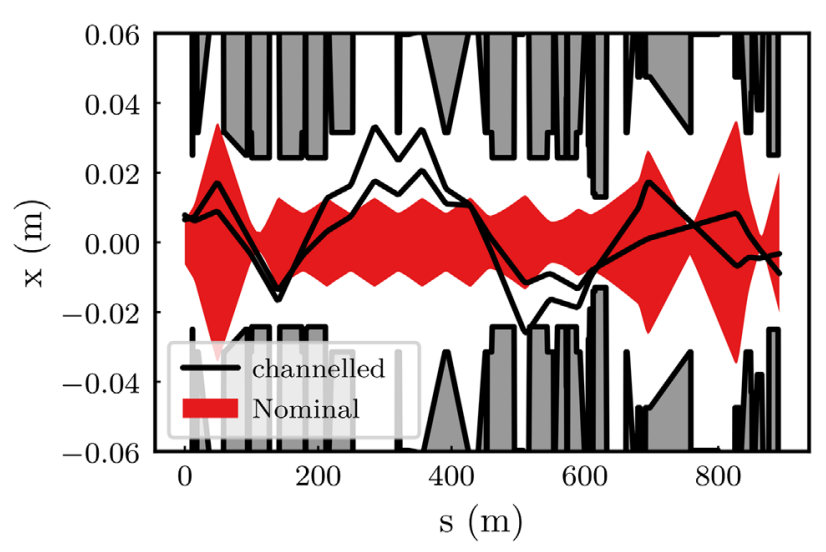

FIG. 18. Envelope of the extracted beam and channeled beamlet transported to the target and the envelope of the channeled beamlet.

shadowing on the operational cycle. The nonlocal scenario, as mentioned before and shown in simulations, should lead to the possibility to reduce the phase-space area occupied by the extracted beam and simplify the optics requirements for TT20.

\section{B. Stability and reproducibility}

One of the main concerns for the feasibility of the crystal shadowing concept with a resonantly extracted beam was the machine stability required to hold the separatrix angle at the crystal inside the very limited channeling angular acceptance of the crystal. This was even more challenging on a pulsed, cycling mode needed for the slow extraction cycle. Previous beam tests with crystal channeling in the SPS have only been carried out in a coasting mode where the beam is ramped to approximately $270 \mathrm{GeV}$ and held in static conditions for many minutes or hours as the crystal is actuated and aligned to the beam. The stability and reproducibility are important operational aspects especially in view of the increasingly frequent changes in the SPS cycle composition that introduce hysterisis effects that perturb the beam.

It was apparent from the first tests that channeling and loss reduction could be achieved consistently with remarkable stability. The stability was tested by holding the crystal fixed for a large number of cycles in channeling and volume reflection, with an RMS stability of $1.1 \%$ and $0.4 \%$, respectively, as shown in Fig. 19. A stability check was also made at an angle between channeling and amorphous scattering where the slope of the loss with angle was steepest. Even in this case the stability was fully acceptable.

Slow drifts over longer timescales are not excluded, but a simple automatic algorithm to optimize the crystal angle could be implemented, triggered either on demand or when the normalized losses become too high. More problematic are the changes in loss reduction when the SPS cycle composition changes. Efforts are underway to understand

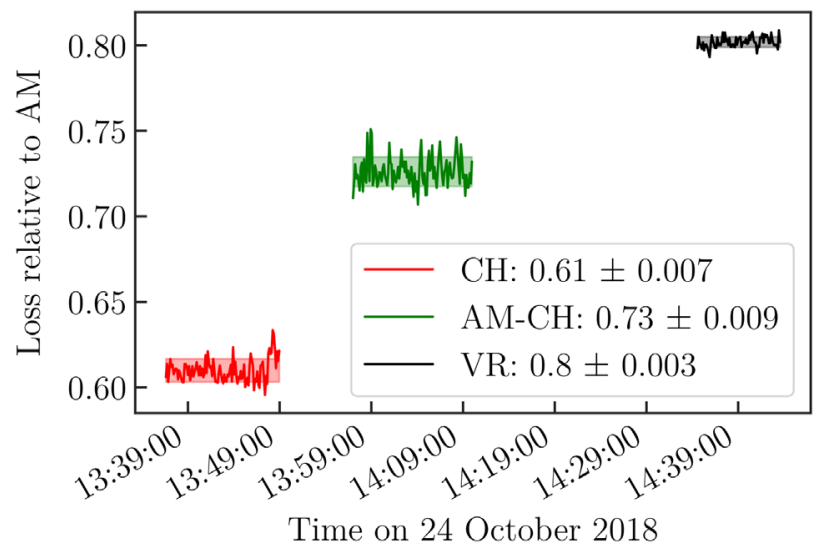

FIG. 19. Beam loss measured at the ES relative to amorphous (AM) during the stability checks. The crystal orientation was fixed in channeling $(\mathrm{CH})$, in the channeling-amorphous $(\mathrm{CH}-$ $\mathrm{AM}$ ) and volume reflection (VR) regimes. The loss reduction factor and RMS are indicated in the legend.

and improve the stability of the SPS as its magnetic cycle is changed in order to mitigate such issues at the source [22].

Another major concern was whether the channeling angle could be maintained throughout the duration of the spill as the tune is swept. This concern motivated the development and operational implementation of COSE. The crystal provided a unique opportunity to probe the efficacy of COSE and the beam's separatrix presentation to the crystal by using loss response of the time resolved LHC and SPS-type BLMs. As a result, it was validated that the loss reduction was indeed maintained throughout the spill. In addition, the LHC BLM next to the crystal also showed the beam remained in channeling throughout the spill. In Fig. 20, the time evolution of the loss reduction at the ES is plotted during the angular scan previously presented in Fig. 14. As the loss reduction during shadowing is independent of time, one concludes that, at least on the

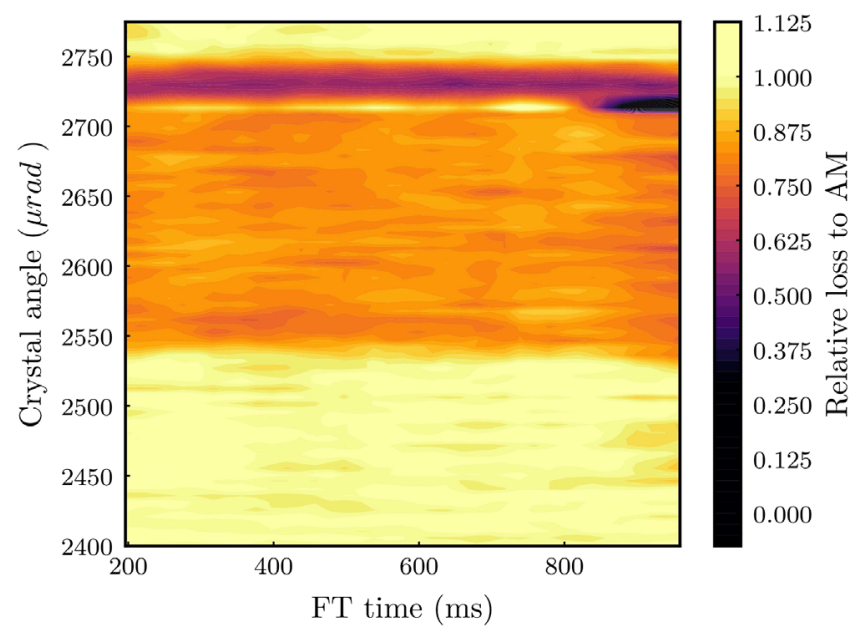

FIG. 20. Time evolution of the loss reduction at the ES measured on the SPS BLM system plotted during the angular scan presented in Fig. 14. 


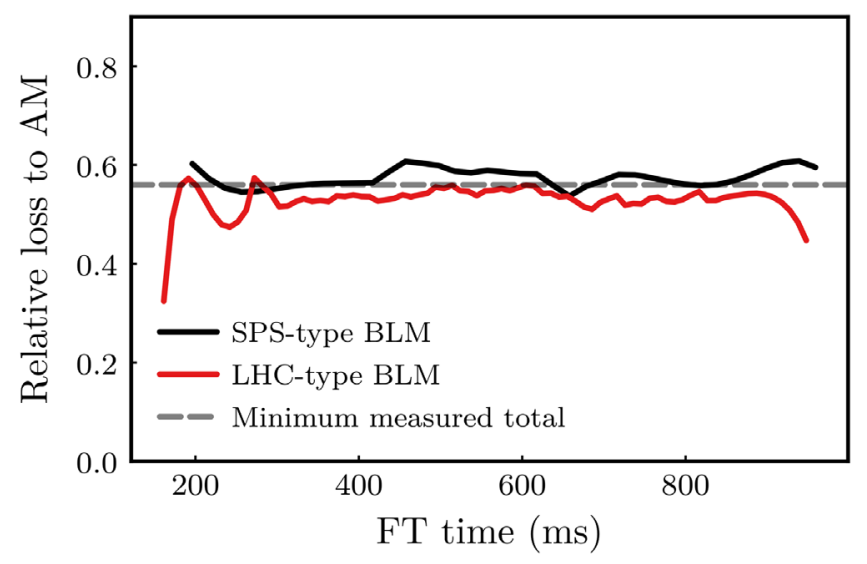

FIG. 21. Time evolution of the loss reduction for the optimized case in channeling presented in Fig. 14.

level of only a few $\mu \mathrm{rad}$, the separatrix does not move during the spill. This is emphasized in Fig. 21, where the beam loss reduction is plotted as a function of time for the optimized case in channeling previously presented in Fig. 14. The result is confirmed on the independent LHCtype and SPS-type BLM systems. The dynamics of the beam loss during the spill with and without COSE optics is discussed in more detail in [9].

\section{Deployment of the system on the operational beam}

The crystal was also deployed for a $13 \mathrm{~h}$ period on the operational beam to the North Area experiments, with a high intensity of $2.8 \times 10^{13}$ protons per spill. A total of $\sim 6 \times 10^{16}$ protons were extracted during this time with approximately $5 \%$ of this intensity estimated to have directly impinged the crystal. For simplicity, it was decided to test the shadowing concept in volume reflection due to concerns of causing downtime to operation from losing the channeled beamlet on an aperture restriction in TT20 with a different transfer line optics that was used during the BDF prototype target tests. The crystal was inserted and its angle set to VR with the settings found in earlier tests. The setup took no more than $10 \mathrm{~min}$ of downtime. A negligible amount of outgassing was observed when the crystal was inserted, which was an order of magnitude lower than what observed for the passive diffuser.

The expected inelastic scattering probability, which dominates the induced radioactivity of the crystal after particle-material interaction, was measured in the order of $0.5 \%$ when oriented in amorphous, for a $1.94 \mathrm{~mm}$ long crystal [23]. During the test on the operational beam, the absolute prompt dose level observed at the location of the crystal increased by an amount comparable to $1 \%$ of the total dose saved around the SPS ring. This value corresponds to $0.2 \%$ of the total particles expected to have impinged on the crystal, which is reasonable agreement with the measurements presented in [23].

No issues were experienced during the test and a $20 \%$ loss reduction was observed throughout, as expected. There was also strong evidence that the extracted beam intensity was boosted with the crystal inserted.

\section{OPERATIONAL PERFORMANCE REACH}

The experimental results from the SPS have identified clear directions for further optimization of the system. The ES width that best fits the observed results is $0.5 \mathrm{~mm}$ and as a result the optimum crystal width is $0.8 \mathrm{~mm}$ for the local case. Although this is the width of the installed crystal, the channeling angle could be reduced to $120 \mu \mathrm{rad}$, which should slightly increase the channeling efficiency and beamloss reduction.

To further reduce losses at the ES in local mode, an array of precisely aligned crystals placed in VR to optimize the efficiency could be envisaged. In this configuration, the sensitivity to separatrix angle variations would be reduced due to the large available angular acceptance of VR.

The nonlocal case could represent an approach to reduce the beam loss at the ES by a factor of four using a single crystal in channeling. Simulations have shown great potential for such a concept, although the operational complications of tuning two separate closed-orbit bumps has to be evaluated with beam tests.

Finally, the combination of the crystal shadowing with separatrix density manipulation with octupoles could bring an additional factor 1.5 to 2 gain.

\section{A. Crystal operation with high intensity}

It is shown in literature [24-27] that exposure to high intensity beams could affect the crystal properties, for example deflection efficiency. For the proposed concept, the crystal in the SPS was already exposed, and will be exposed in the future, to approximately $8 \%$ and $18 \%$, for the local and nonlocal shadowing case respectively, of the extracted intensity. This corresponds to about $5 \times 10^{12} \mathrm{p} /$ cycle impinging the crystal in the case of high intensity extraction expected for SHiP operation. As the target POT for SHiP era is $4 \times 10^{19} \mathrm{POT} /$ year and the irradiated area is in the order of 3.2 and $7.2 \mathrm{~mm}^{2}$, it is expected to deliver approximately $1 \times 10^{18} \mathrm{p} / \mathrm{mm}^{2}$ in one year on the crystal, for both cases. As shown in [26], irradiation with $2.4 \times$ $10^{20} \mathrm{p} / \mathrm{cm}^{2}$ at $450 \mathrm{GeV}$ produced a local reduction in channeling efficiency of about $30 \%$. Although the expected delivered intensity to the crystal for ES shadowing seems significantly smaller, the crystal surface that will be impinged with protons is much smaller, hence it is difficult to fully conclude on the expected long-term performances of the crystal in the SPS. This will be undoubtedly one of the answers that will be sought during the restart of the SPS in 2021.

Moreover, in the unlikely case of observation of reduction in performance or any misbehavior of the crystal or the goniometer, exchange can be foreseen at the end of the operational year. 


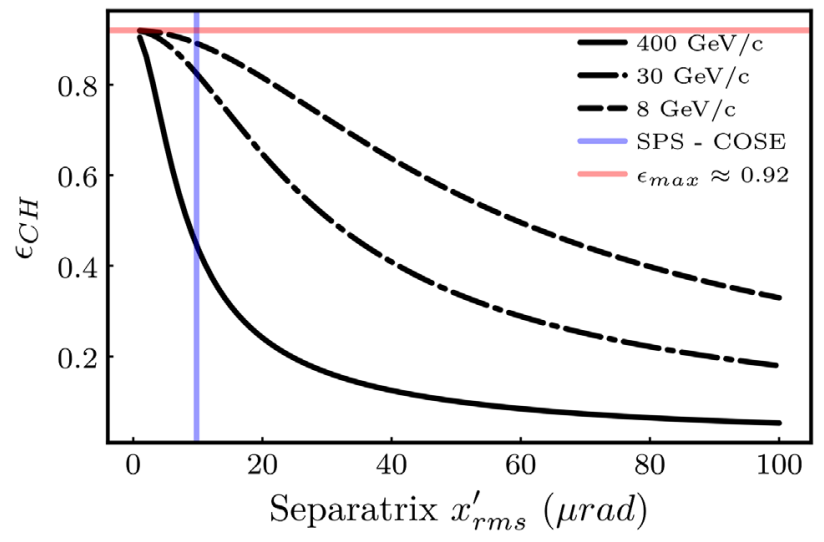

FIG. 22. Channelling efficiency for the crystal used in the SPS test as a function of the separatrix RMS angular spread. The dependence has been parametrized for three different beam energy: 8,30 , and $400 \mathrm{GeV} / \mathrm{c}$.

\section{APPLICATION AT DIFFERENT ENERGY EXTRACTION}

The channeling angular acceptance depends on the impinging particle momentum, as the crystal critical angle is proportional to $1 / \sqrt{p v}$ [25], where $p$ is the particle momentum and $v$ the velocity. The maximum efficiency in channeling $\left(\epsilon_{\max }\right)$ is only weakly dependent on particle momentum [25]. Since the separatrix angular spread $(1 \sigma)$ at the ES mainly depends on the extraction methodology and the instantaneous momentum spread, this means that crystal shadowing will be more effective at lower energies.

The fraction of impinging particles channeled $\left(\epsilon_{\mathrm{CH}}\right)$ as a function of separatrix angular spread for different energies is shown in Fig. 22. It is interesting to note how the efficiency remains high for large angular spread for lower energy beams. Detailed simulations are needed to evaluate the total loss reduction with the crystal shadowing for lower energies, since the overall effect depends also on multiple Coulomb scattering which is inversely proportional to the particle momentum, while space charge and other effects specific to the extraction method contribute to the total separatrix width. In fact, the high efficiency that can be achieved for low energy beams relies on the usage of techniques to maintain the total angular spread at the crystal as low as possible, e.g., COSE or dynamic bump.

\section{CONCLUSIONS AND OUTLOOK}

A new method of substantially reducing the beam loss inherent in the slow extraction process has been developed and tested, exploiting silicon bent crystal technology to coherently deflect the part of the beam that would otherwise strike the blade of the extraction septum. The shadowing of the extraction septum blade with a bent crystal was shown in machine studies to reduce $400 \mathrm{GeV}$ proton beam lost on the SPS electrostatic extraction septum by over $40 \%$, and further optimisation of the crystal width, bend angle and location in the machine should allow more than double this number to be achieved by this method alone. Operational aspects including the methods needed to set-up and optimize the new extraction scheme were tested and the stability of the loss reduction with time was measured. The parallel development of a new COSE extraction method at the SPS allows the required separatrix angular stability and spread to be maintained throughout the spill. Future challenges remain, including the control of the longer term machine stability as the magnetic cycle of the SPS is changed at a greater frequency to accommodate different users in its growing physics programme. This issue will need to be addressed, either by reducing the sensitivity to such changes, by reducing the separatrix angular thickness, or by a feed-forward system to correct for the changes in a dynamic manner.

The technique is of general interest for existing high power proton accelerators and for future machine designs. For the SPS, the possibility of increasing the extraction efficiency by a factor of four is a major opportunity, both for improving the lifetime and maintainability of the present accelerator systems and also for increasing the protons extracted per year for CERN's future physics programs.

\section{ACKNOWLEDGMENTS}

The authors would like to express their gratitude to the UA9 collaboration for their support and their contribution to the development of the experimental apparatus used in this study. The authors would also like to thank the SPS operation, beam instrumentation, septa and rf teams for their support, as well as the Experimental Areas, Physics and Machine Development coordinators for their flexibility and understanding in the scheduling of the beam tests.

[1] M. A. Fraser et al., SPS Slow Extraction Losses and Activation: Challenges and Possibilities for Improvement, in Proc. of IPAC'17, paper MOPIK045, Copenhagen, Denmark, 2017, pp. 631-634, http://accelconf.web.cern .ch/AccelConf/ipac2017/papers/mopik045.pdf.

[2] M. A. Fraser et al., SPS Slow Extraction Losses and Activation: Update on Recent Improvements, in Proceedings of 10th Int. Partile Accelerator Conf. IPAC2019, Melbourne, Australia (JACoW Publishing, Geneva, Switzerland, 2019), pp. 2391-2394.

[3] M. A. Fraser et al., Slow Extraction Efficiency Measurements at the CERN SPS, Proceedings of 9th International Particle Accelerator Conference IPAC'18, Vancouver, BC, Canada, 2018 (JACoW Publishing, Geneva, Switzerland, 2018), paper TUPAF054.

[4] A. A. Asseev and M. Yu. Gorin, One more possibility of using bent crystals for beam extraction from accelerators, Nucl. Instrum. Methods Phys. Res., Sect. A 372, 1 (1996). 
[5] A. G. Afonin et al., Implementation of multistrip crystals to protect the septum magnets and to generate gamma radiation, Phys. Rev. Accel. Beams 22, 033001 (2019).

[6] F. M. Velotti, Ph.D. thesis, Higher brightness beams from the SPS for the HL-LHC era, Ecole Polytechnique de Lausanne Report No. CERN-THESIS-2017-041, 2017.

[7] A. Golutvin et al., A facility to search for hidden particles (SHiP) at the CERN SPS, CERN Reports No. CERNSPSC-2015-016 (No. SPSC-P-350), 2015.

[8] M. Shaposhnikov, The $\nu$ MSM, leptonic asymmetries, and properties of singlet fermions, J. High Energy Phys. 08 (2008) 008.

[9] V. Kain, F. M. Velotti, M. A. Fraser, B. Goddard, J. Prieto, L. S. Stoel, and M. Pari, Resonant slow extraction with constant optics for improved separatrix control at the extraction septum, Phys. Rev. Accel. Beams (to be published).

[10] R. Rossi, G. Cavoto, D. Mirarchi, S. Redaelli, and W. Scandale, Measurements of coherent interactions of $400 \mathrm{GeV}$ protons in silicon bent crystals, Nucl. Instrum. Methods Phys. Res., Sect. B 355, 369 (2015).

[11] M. Tomizawa et al., Performance of resonant slow extraction from J-PARC Main Ring, in Proceedings of the 3rd International Particle Accelerator Conference, New Orleans, LA, 2012 (IEEE, Piscataway, NJ, 2012), paper MOPPD051, pp. 481-483.

[12] L. S. Stoel et al., Progress toward a dynamic extraction bump for slow extraction in the CERN SPS, in Proceedings of 9th International Particle Accelerator Conference IPAC18, Vancouver, BC, Canada, 2018 (JACoW Publishing, Geneva, Switzerland, 2018), paper TUPAF055, pp. 838-841.

[13] M. Albert et al., LHC Software Architecture: TI8 commissioning, CERN Report No. CERN-LHC-Project-Note368, 2005.

[14] W. Scandale et al., First results on the SPS beam collimation with bent crystals, Phys. Lett. B 692, 78 (2010).

[15] W. Scandale et al., Comparative results on collimation of the SPS beam of protons and $\mathrm{Pb}$ ions with bent crystals, Phys. Lett. B 703, 547 (2011).

[16] W. Scandale et al., Strong reduction of the off-momentum halo in crystal assisted collimation of the SPS beam, Phys. Lett. B 714, 231 (2012).
[17] Methodical Accelerator Design https://madx.web.cern.ch.

[18] P. K. Skowronski, E. Forest, and F. Schmidt, Advances in MAD-X using PTC, Report No. CERN-LHC-PROJECTReport-1016, https://cds.cern.ch/record/1056684.

[19] L. Esposito et al., Crystal for Slow Extraction LossReduction of the SPS Electrostatic Septum, in Proceedings of 10th Int. Particle Accelerator Conf. IPAC'19, Melbourne, Australia, 2019 (JACoW Publishing, Geneva, Switzerland, 2019), paper WEPMP028.

[20] B. Goddard et al., Reduction of $400 \mathrm{GeV}$ slow extraction beam loss with a wire diffuser at the CERN SPS (to be published).

[21] M. A. Fraser, B. Goddard, and F. M. Velotti, Functional specification for a thin bent crystal located in LSS2 for the shadowing tests of the ZS wires in 2018, CERN Report No. EDMS no. 1783433, 2018.

[22] F. M. Velotti et al., Observations of SPS Slow-Extracted Spill Quality Degradation and Possible Improvements, Proceedings of 9th International Particle Accelerator Conference IPAC'18, Vancouver, BC, Canada, 2018 (JACoW Publishing, Geneva, Switzerland, 2018), paper TUPAF035.

[23] W. Scandale et al., Probability of inelastic nuclear interactions of high-energy protons in a bent crystal, Nucl. Instrum. Methods Phys. Res., Sect. B, 268, 2655 (2010).

[24] V. M. Biryukov et al., Crystal Channeling and its Application at High-Energy Accelerators (Springer, New York, 1997).

[25] A. Baurichter et al., Channeling of high-energy particles in bent crystals-Experiments at the CERN SPS, Nucl. Instrum. Methods Phys. Res., Sect. B 164-165, 27 (2000).

[26] C. Biino et al., The influence of radiation damage on the deflection of high energy beams in bent-silicon crystals, Report No. CERN-SL-96-030-EA, European Particle Accelerator Conference, pp. e-proc. 2385-2387, Sitges, Barcelona, Spain, 1996, http://accelconf.web.cern.ch/Accel Conf/e96/PAPERS/MOPG/MOP014G.PDF.

[27] S. I. Baker, R. A. Carrigan, V. R. Cupps, J. S. Forster, W. M. Gibson, and C.R. Sun, Effects on channeling of radiation damage due to $28 \mathrm{GeV}$ protons, Nucl. Instrum. Methods Phys. Res., Sect. B 90, 119 (1994). 